Ebisu Ebisu

Études japonaises Études japonaises

$54 \mid 2017$

L'après-guerre des intellectuels japonais

\title{
Philippe Pons, Corée du Nord. Un État-guérilla en
}

mutation

Paris, coll. « La suite des temps », Gallimard, 2015, 707 pages

Patrick Fridenson

\section{OpenEdition}

Journals

Édition électronique

URL : http://journals.openedition.org/ebisu/2148

DOI : 10.4000/ebisu.2148

ISSN : 2189-1893

Éditeur

Institut français de recherche sur le Japon à la Maison franco-japonaise (UMIFRE 19 MEAE-CNRS)

Édition imprimée

Date de publication : 19 décembre 2017

Pagination : 271-274

ISSN : $1340-3656$

Référence électronique

Patrick Fridenson, «Philippe Pons, Corée du Nord. Un État-guérilla en mutation », Ebisu [En ligne], 54

2017, mis en ligne le 19 décembre 2017, consulté le 23 septembre 2020. URL : http://

journals.openedition.org/ebisu/2148; DOl : https://doi.org/10.4000/ebisu.2148 
Livres à lire 

d'exception culturelle. On pourrait continuer ainsi, en suggérant de nombreuses autres pistes, avec parallélismes, échos et oppositions, pour traverser cette anthologie riche, soignée et intelligente: un instrument qu'on peut envier à nos voisins.

Nicolas MolLard Univ. Jean Moulin Lyon 3

1. Notamment dans " Thinking with the Past: History Writing in Modern Japan ", in Wm. Theodore de Bary, Carol Gluck, and Arthur Tiedemann (eds.), Sources of Japanese Tradition, vol. 2, $2^{\mathrm{e}}$ édition, New York, Columbia University Press, 2006.

() Philippe Pons, Corée du Nord. Un État-guérilla en mutation, Paris, coll. " La suite des temps ", Gallimard, 2015, 707 pages.

Des missiles programmés par Pyongyang menacent régulièrement le Japon. La Corée du Nord défraye la chronique chaque mois. Si elle fait l'objet d'articles dans des revues scientifiques françaises ${ }^{1}$, il n'y avait pourtant pas en français d'ouvrage d'ensemble à son sujet. Or, de nombreuses études ont été produites par des chercheurs de pays directement concernés: Chine, Japon,
Corée du Sud, Russie, ainsi que par des Américains, et "beaucoup moins ", par des Européens. Cette absence d'ouvrage de référence en français est désormais comblée. Le livre de Philippe Pons est d'abord une somme, fruit de nombreuses années de travail. Le lecteur appréciera par exemple les pages 301-304 sur l'utilisation par le régime nordiste de Macao comme tête de pont de tous les trafics possibles, illicites mais aussi licites. Comme non-spécialiste de la question, je pourrais m'en réjouir, et ajouter que la simplicité de son plan chronologique en trois parties: «l'ère Kim Il Sung », « l'ère Kim Jong $\mathrm{Il}$ » et "Kim, troisième du nom » aide à la lisibilité de l'ouvrage. Mais ce n'est pas la principale vertu du livre, laquelle, comme toute bonne recherche, est d'avoir une thèse.

Cette thèse est en vérité double. (1) $\mathrm{Si}$ le régime totalitaire nordcoréen est un État-guérilla, concept que Philippe Pons emprunte à l'historien japonais Wada Haruki, soit un État qui organise la population sur le modèle d'une unité de guérilla et entretient une mentalité d'assiégé permanent chez ses sujets, sa longévité s'explique principalement par la force du nationalisme que partage la population et que cultive le régime lui-même. Ce nationalisme puise ses racines dans l'expérience de la colonisation japonaise, du joug japonais. 
Pour l'entretenir, le communisme successoral au pouvoir prend appui sur l'éventualité d'un recours à l'arme nucléaire brandie périodiquement par les dirigeants américains depuis 50 ans, à l'exclusion du président Obama. Il me semble qu'on retrouve ici un type d'explication majeur que le politiste américain Chalmers Johnson avait lancé dans son premier livre, en 1963, pour comprendre l'enracinement du communisme en Yougoslavie comme en Chine. Cependant trois composantes propres à la Corée du Nord sont soulignées par Philippe Pons. La première est l'influence d'un christianisme implanté dans des couches bourgeoises, Pyongyang ayant été la ville la plus chrétienne d'Asie après Manille. On relève en particulier le rôle du protestantisme dans ce nationalisme, et on sait le ferment de critique sociale et de contestation qu'il a apporté ensuite en Corée du Sud jusqu'à aujourd'hui. La seconde composante est que le Nord avait, à la partition, un niveau de développement industriel et une faible proportion d'agriculteurs qui évoquent plus certains pays d'Europe de l'Est que les pays analysés par Chalmers Johnson : donc aujourd'hui ce «n'est pas un pays pauvre mais un pays ruiné » (p. 649). La troisième est que la part des communistes dans la libération de la Corée est bien moindre qu'en Yougoslavie ou en Chine. (2) L'histoire ne s'arrête pas aux origines. Elle n'est pas figée ici plus qu'ailleurs. La permanence de l'Étatguérilla et de son aventurisme belliqueux, qui alimente la propagande architecturale, picturale, télévisuelle et cinématographique (p. 255-291), ne doit pas dissimuler une autre réalité profonde : le peuple de la RPDC «ne forme pas une masse amorphe et résignée, composée de robots décervelés, de bourreaux ou de victimes » (p. 31 et 650). Il convient donc, tant pour les États du reste du monde que pour les chercheurs, de ne pas s'en tenir aux facilités intellectuelles et politiques de la diabolisation, de prendre au sérieux la volonté de changement d'une grande partie de la population face à son "tragique destin ", d'accompagner les efforts de celles et ceux qui, à différents niveaux, œuvrent à remettre en marche la société, l'économie, la culture, notamment à partir des activités ou des débats qui en sont des indices.

Il n'est pas possible ici d'évoquer toutes les richesses de ce livre. Je me limiterai à deux points.

Le premier, nul n'en sera surpris, est le traitement très informé de la question nucléaire. Philippe Pons suit le passage du nucléaire civil au nucléaire militaire en soulignant (p. 304 et 306) les deux globalisations successives qu'il a impliquées pour 
la Corée du Nord. «Le programme nucléaire nord-coréen a connu quatre phases : initiation par les Soviétiques (années 1950); obtention par des moyens détournés de savoir-faire et de technologies (1960-1970); mise en place progressive d'une filière indépendante (1970-1990); et maturation du programme ». Si la première phase est civile, appuyée par les Soviétiques et destinée à créer une industrie d'usage civil de l'énergie nucléaire, en revanche la formation d'une filière militaire se heurte à l'opposition des Soviétiques et est réalisée avec d'autres types de partenaires étrangers : " avec la technologie civile soviétique, puis en débauchant des ingénieurs de l'ex-URSS et en se fournissant au Pakistan en technologie et en équipements (dont une partie fut aussi acquise secrètement au Japon) ». En revanche, c'est une approche géopolitique que Philippe Pons adopte lorsqu'il traite plus tard de la «deuxième crise nucléaire » (p. 486-500).

Le second point concerne l'entrée dans l'économie de marché. L'historien économiste que je suis a lu les pages correspondantes, et notamment le chapitre 14 et dernier, avec la plus grande attention. La prise de conscience par un nombre croissant de décideurs, notamment à la suite de la grande famine, du carcan d'un système "collectiviste et hypercentralisé » est analysée et, au-delà du nouveau discours officiel sur le collectivisme «flexible et responsable ", l'évolution vers une économie hybride articulant le public et le privé est cernée par l'auteur, permettant des rapprochements avec la trajectoire suivie par d'autres pays communistes, la Chine en tête.

Philippe Pons est à la fois, aux côtés de Christian Sautter, le cofondateur du Centre de recherche sur le Japon contemporain de l'École des hautes études en 1973 et un journaliste spécialisé dans la couverture du Japon et de la Corée. Cette double caractéristique, fort précieuse et fort rare, explique la variété des sources qui forment la base de ce livre qui fera date. Depuis le Congrès du PC de 1980 l'auteur s'est rendu treize fois en Corée du Nord pour enquêter sur place, ce qui n'a pas été chose facile dans un pays aussi verrouillé, où il s'agit par exemple de faire croire que l'on rencontre des passagers au hasard quand l'on est dans un wagon de métro. Mais la vigilance et le sens critique ne sont pas les seules qualités nécessaires. L'auteur a aussi été à l'écoute de la "connaissance du terrain" accumulée par les ONG françaises "présentes depuis de nombreuses années " dans le pays. Il a su inspirer "confiance " à des Coréens du Nord rencontrés dans d'autres pays comme sur place, ainsi qu'à des réfugiés. Il a bénéficié de rencontres 
avec différents chercheurs étrangers. Il a lu une partie des innombrables sources imprimées produites par le régime dans sa langue de bois spécifique. Il a mobilisé les travaux scientifiques disponibles en japonais et en anglais. Il ne s'est jamais enfermé dans une approche pointue et Leo Strauss, Fernand Braudel, Roger Chartier et d'autres ont été consultés pour éclairer la route.

Il faut donc remercier Pierre Nora d'avoir donné à l'auteur toute la place nécessaire en l'accueillant dans sa collection "La suite des temps". On a ici un grand livre, où l'auteur fait preuve d'un vrai bonheur d'écriture. On y trouve des péripéties et des personnalités dignes des pièces de Shakespeare. On y trouve aussi la monstruosité et l'impéritie que, dans un livre récent chez le même éditeur (que cite $\mathrm{Ph}$. Pons) La récidive : révolution russe, révolution chinoise (Paris, Gallimard, 2014), l'historien Lucien Bianco montrait chez Staline et chez Mao, et dès lors on peut parler d'une nouvelle "récidive" de la tyrannie. On aimerait en savoir plus sur les cadres de l'armée et sur les universitaires qui ont grandi dans le régime, car ils détiennent quelques clés du présent et de l'avenir. Bien sûr, on sait par avance qu'il existe dans la coréanologie internationale des chercheurs qui ont des interprétations opposées à celles de ce livre et qui le diront.
Il n'empêche : derrière la nécessairement minutieuse histoire des cliques et des crimes, des missiles et des provocations en tous genres, de la police des pensées et du contrôle social, le tout sous l'œil de la Chine, on sait énormément gré à Philippe Pons d'avoir cherché, pour chaque étape de cette histoire, à nous faire voir dans ce livre le peuple, qui souffre, que le creusement des inégalités et l'essor de la corruption inquiètent et qui, de par les capacités nouvelles qui sont en train de se créer, peut avoir d'autres horizons que les élites au pouvoir.

\section{Patrick Fridenson} EHESS

1. Et encore peuvent-ils être l'œuvre de chercheurs étrangers. Cf. Hazel Smith, « La Corée du Nord en route vers l'économie de marché ", Critique internationale, $\mathrm{n}^{\circ} 15$, 2002, p. 6-14 (non cité dans la bibliographie du livre parmi les travaux de cet auteur). 